\title{
THE EFFECT OF THE ADDITION OF ACTIVATED CHARCOAL ON THE ANTIBACTERIAL EFFICIENCY OF DIFFERENT CALCIUM HYDROXIDE FORMULATIONS
}

\author{
Medhat Taha Elfaramawy*, Shady Ali Hussein* and Nesma AbdelAziz Hamdi**
}

\begin{abstract}
Aim of the study: Is to compare the antibacterial effect of conventional calcium hydroxide (GAMA dental lab, Cairo, Egypt), calcium hydroxide with Activated Charcoal (Eucarbon, Sedico, Cairo, Egypt), calcium hydroxide paste with iodoform (Metapex) (META,Chungcheongbukdo,Korea ) and calcium hydroxide paste with iodoform (Metapex) mixed with Activated Charcoal against E-faecalis using Agar diffusion method.
\end{abstract}

Materials and Methods: Samples were classified into 4 groups according to the material used. Each group was further divided into 3 subgroups according to the observation time ( 1 day, 3 days and 7 days). Each subgroup consisted of 7 agar plates implanted with E-faecalis strain. Four Holes were created in each agar plate each hole contained one tested material.

Results: Conventional Calcium hydroxide with saline had the largest inhibitory zone of $\mathrm{E}$ faecalis in agar diffusion test over all observation period followed by Activated Charcoal and conventional calcium hydroxide followed by calcium hydroxide paste with iodoform with Activated Charcoal and the calcium hydroxide mixed with iodoform had no change in inhibitory zone.

Conclusion: The addition of activated charcoal did not improve the antibacterial effect of conventional calcium hydroxide while it improves the antibacterial efficacy of the calcium hydroxide and iodoform (Metapex).

\section{INTRODUCTION}

Chemical and biological dynamics of any intra canal medication is an important issue for choosing the desired intra canal medication. Microorganisms infecting the root canal system have a limited virulence when it is acting as an individual species how- ever it is of high virulence when acting collectively. The success of endodontic treatment is highly dependent on the elimination of the infection from the root canal system, different intra canal medications has been used for this purpose however still the calcium hydroxide is considered the most popular and efficient intra canal medication used ${ }^{(1-5)}$. So many

\footnotetext{
* Associate Professor, Endodontic Dep., Faculty of Dentistry, Ainshams University.

** Department of Microbiology. Faculty of Medicine. Ain Shams University
} 
changes were done to increase the antimicrobial action of calcium hydroxide that can speed up or slow down the ionic dissociation such as changing of the vehicle, addition of other active ingredients such as iodoform or changing of the particle size with use of the Nanotechnology, and adding different materials that may improve the antibacterial effect of calcium hydroxide formulations. The purpose of this study was to evaluate the effect of the addition of activated charcoal on the antibacterial efficiency of different calcium hydroxide formulations.

\section{MATERIALS AND METHODS}

\section{Material}

In this study, the following materials were used

\section{a) Bacterial isolates}

A total of (21) E-faecalis isolates were included in this study. All E-faecalis strains used in this study were isolated from human stool samples by culturing on MacConkey agar medium followed by subculture on nutrient agar medium for purification of the isolated colonies ${ }^{(9)}$.

\section{b) Substances:}

- Calcium hydroxide Powder with saline.

- Calcium hydroxide with saline+ Activated Charcoal conventional calcium hydroxide .

- Calcium hydroxide past with iodoform.

- Calcium hydroxide past with iodoform + Activated Charcoal.

\section{c) Media:}

The following media were used in this study:

\section{Brain-Heart Infusion Broth:}

This medium was used to prepare the suspension of E-faecalis.

\section{Brain-Heart Infusion Agar}

This medium was used to test the effect of the different substances on the growth of E-faecalis using the diffusion agar method.

\section{Methods}

\section{a) Preparation of Brain-Heart Infusion Broth: ${ }^{(7)}$}

Thirty-seven grams of the medium were suspended in one liter of distilled water. Heating with frequent agitation ensured good mixing \& dissolution. The suspension was then boiled for one minute until complete dissolution. It was later dispensed into appropriate containers and sterilized at $121^{\circ} \mathrm{C}$ for 15 minutes. The prepared medium was stored at $2-8^{\circ} \mathrm{C}$. For best results, the medium was used on the same day.

\section{b) Preparation of Brain-Heart Infusion Agar: ${ }^{(7)}$}

Fifteen grams of agar powder were added to 1 liter BHI broth \& then heated to dissolve agar before dispensing into appropriate containers. Autoclaving was then performed for $15 \mathrm{~min}$ at $121^{\circ} \mathrm{C}$ to ensure adequate sterilization. The mixture was then poured into Petri dishes \& left to cool \& solidify.

\section{c) Preparation of E-faecalis Suspension:}

A sterile swab was used to transfer bacterial growth from the primary culture into the BHI broth bottle \& mixed well to form a homogenous suspension.

\section{d) Classification of samples:}

21 samples were classified according to the tested material into 3 groups;

Group 1: Consisted of 7 holes filled with Calcium hydroxide Powder

Group 2: Consisted of 7 holes filled with Calcium hydroxide with saline+ Activated Charcoal conventional calcium hydroxide

Group 3: Consisted of 7 holes filled with calcium hydroxide paste with iodoform 
Group 4: Consisted of 7 holes filled with calcium hydroxide paste with iodoform + Activated Charcoal

Each group was further divided into 3 subgroups according to the observation period to;

Subgroup A: One day.

Subgroup B: Three days.

Subgroup C: Seven days.

\section{e) Implantation of the E-faecalis in the agar plates}

A sterile cotton swab was dipped into the suspension and excess fluid was removed by turning the swab against the inside of the tube. The inoculum was evenly spread over the entire surface of dry BHI agar plates by swabbing in three different directions ${ }^{(8)}$.

\section{f) Mixing and application of the tested substances}

Three holes of $4 \mathrm{~mm}$ diameter were made on the agar surface by a metal punch leaving about 10-15 $\mathrm{mm}$ away from the edge of the petri dish, and these holes were separated from each other by a distance not less than $20 \mathrm{~mm}$ to avoid overlapping zones of inhibition. Each hole contained one of the tested materials and marked as following;

- Hole number I for conventional Calcium hydroxide Powder.

- Hole number II for conventional Calcium hydroxide with Activated Charcoal.

- Hole number III for calcium hydroxide past with iodoform. and

- Hole number IV for calcium hydroxide past with iodoform mixed with Activated Charcoal.

All the agar plates were incubated at $37^{\circ} \mathrm{C}$ in aerobic conditions for the required observation periods

\section{g) Method of evaluation}

After one day observation the area of microbial growth inhibition (lack of bacterial colonization) around the holes were measured at the largest diameter with a poly gauge millimeter ruler. The agar plates were then re-incubated to take the measurements of the inhibitory zones after one day, three days and then after 7 days

\section{h) Statistical analysis}

Data were analyzed by SPSS software (version 16.0, SPSS, Chicago, IL, USA). Data in each group were compared by the ANOVA and Kruskal-Wallis tests. Also the Dunnett's test was performed to compare the results between two groups. The level of significance was set at 0.05 .

\section{RESULTS}

\section{A) Group one (Conventional Calcium hydroxide} Powder)

The inhibitory zone after one day or 3 days or 7 days was the same changed to $22 \mathrm{~mm}$

\section{B) Group two (Conventional Calcium hydroxide Powder + Activated Charcoal)}

The inhibitory zone after one day or 3 days or 7 days was the same changed to $21 \mathrm{~mm}$

\section{C) Group three (calcium hydroxidepaste with io- doform)}

There is no change of the inhibitory zone after one day or 3 days or 7 days

\section{D) Group four (calcium hydroxide past with iodo- form +Activated Charcoal):}

The inhibitory zone after one day or 3 days or 7 days was the same changed to $16 \mathrm{~mm}$

\section{DISCUSSION}

The main purpose of endodontic treatment is the elimination of micobiota from the root canal system, which is the main challenge for all the endodontic practitioners, sporadic species of bacteria affecting the root canal system has low virulence impact 
however collectively they have high virulence impact ${ }^{(9,10)}$. Estrela et al $\left({ }^{(1)}\right.$ Suggested the hypothesis of an irreversible inactivation of bacterial enzymes under extreme condition of $\mathrm{PH}$ for long period of time and also a temporary bacterial enzymatic inactivation with the restoration of normal activity when the PH returns to the ideal level. Estrela etal. (3) Suggested the mechanism of action of calcium hydroxide through the release of hydroxyl ions that causing changes in the transport of nutrients and structure of organic component causing bacterial destruction. Lima etal. ${ }^{(11)}$ concluded that all calcium hydroxide medicaments were able to reduce colony forming unit (CFU) values of E-faecalis. Chai et al. ${ }^{(12)}$ also concluded that calcium hydroxide are $100 \%$ effective in eliminating $E$ - faecalis biofilm.

Iodoform is composed of some powder with bright hexagonal crystals, it decomposes releasing iodine in nascent state ${ }^{(13)}$. Compounds containing iodine are very employed for infection control in dentistry and it gives high radio-opacity for calcium hydroxide. The main problem of the action of the calcium hydroxide is that it has to be in direct contact with the bacteria to act on it, that's why the addition of activated charcoal can be of benefit because of its adsorptive action that can attract the bacteria to the site of the calcium hydroxide and so can be beneficial to the antimicrobial effect of calcium hydroxide.

Agar diffusion test is a widely used test with reproducible results for the evaluation of the antimicrobial activity ${ }^{(14)}$. It is able to demonstrate the activity of freshly mixed intra canal medications however it has many limitations as it lacks the ability to test and compare the viability of microorganisms used and the inability to distinguish between the bactericidal and bacteriostatic ability of tested materials ${ }^{(15)}$. The results showed that with conventional calcium hydroxide, the addition of activated charcoal did not improve the inhibitory zone which may be related to the high spread of conventional calcium hydroxide powder mixed with saline. However with the use of calcium hydroxide with iodoform due to its putty consistency it has low spreading action through agar media and so no inhibition zone was detected while with the addition of activated charcoal with its adsorption effect it can attract the bacterial cells to the site of existence of calcium hydroxide and so improves its antibacterial efficacy.

\section{CONCLUSION}

The addition of activated charcoal did not improve the antibacterial effect of conventional calcium hydroxide while it improves the antibacterial efficacy of the calcium hydroxide and iodoform (Metapex).

\section{REFERENCES}

1. Estrela C, Estrela C, Hollanda A, Decurcio A, Pécora J. Influence Of Iodoform On Antimicrobial Potential Of Calcium Hydroxide. J ApplOral Sci. 2006;14(1):33-7

2. Estrela C, MamedeNeto I, Estrela CRA, Pécora JD. Evaluation of density of calcium hydroxide pastes in dog's mandible. BrazEndod J. 1998;3:24-30.

3. Estrela C, Sydney GB, Bammann LL, Felippe O Jr. Mechanism of the action of calcium and hydroxyl ions of calcium hydroxide on tissue and bacteria. Braz Dent J. 1995;6:85-90.

4. Memon NA, Memon MR, Ali F: Assessment of the interappointment pain by using two different intracanal medicaments. Pakistan Oral Dent J. 2013;33(1):145-50.

5. Siqueira JF, Jr, Rôças I, Favieri A, et al. : Incidence of postoperative pain after intracanal procedures based on an antimicrobial strategy. J Endod. 2002;28(6):457-60.

6. Siqueira JF, Jr, Lopes HP: Mechanisms of antimicrobial activity of calcium hydroxide: a critical review. Int Endod J. 1999;32(5):361-9. 10.1046/j.1365-2591.

7. Lúcia Martins Teixeira, Maria Da Glória Siqueira Carvalho, Richard R. Facklam, Patricia Lynn Shewmaker Enterococcus. Manual of clinical microbiology, 11th edition, Volume I, Chapter 23. American Society of Microbiology. doi.10.1128/9781555817381. 
8. Estrela C, Bammann LL, Pimenta FC, Pécora JD. Control of microorganism in vitro by calcium hydroxide pastes. IntEndod J. 2001;34:341-5

9. James H. Jorgensen, John D. Turnidge (2015): Susceptibility Test Methods: Dilution and Disk Diffusion Methods. Manual of Clinical Microbiology, Volume I, Section III, Chapter 71. American Society of Microbiology. doi:10.1128/9781555817381

10. Estrela C, Holland R. Calcium Hydroxide: study based on scientific evidences. J Appl Oral Sci. 2003;11:269-82.

11. Murray P.R., Baron J.H., Pfaller M.A., Jorgensen J.H., and Yolken R.H. Manual of clinical microbiology, $8^{\text {th }}$ edition, American Soceity for Microbiology, Washington D.C.
12. Lima R, Tanomaru J, nior N. \& M. Filho T. Effectiveness of calcium hydroxide-based intracanal medicaments against Enterococcus faecalis. Int. Endod. J, 2012;45:311-316.

13. Chai W.L., Hamimah H., Cheng S. C., Sallam A.A. and Abdullah M. Susceptibility Of Enterococcus Faecalis Biofilm To Antibiotics And Calcium Hydroxide. J. Oral Sci. 2007;49:161-166.

14. Dianat O, Saedi S, Kazem M, Alam M. Antimicrobial Activity of Nanoparticle Calcium Hydroxide against Enterococcus Faecalis: An In Vitro Study. I.E.J. 2015; 10(1):39-43

15. Mazyony S. Batouty K. and Elfaramawy M.T. evaluation of antimicrobial effect of three resin based sealers. Msc. Thesis Endodontic Department Ain Shams Uni. 2016. 